\title{
Disección aórtica aguda
}

\author{
A. MUIÑO MIGUEZ, M.V. VILLALBA GARCÍA, C. LÓPEZ GONZÁLEZ-COBOS, \\ M. GÓMEZ ANTÚNEZ, M. ORTIZ VEGA, J. ORTIZ ALONSO \\ Sección de Medicina de Urgencia. Hospital General Universitario Gregorio Marañón. \\ Madrid.
}

\author{
ACUTE AORTIC DISSECTION
}

\section{RESUMEN}

Objetivo: La disección aórtica aguda es una emergencia médica que se asocia a una elevada mortalidad. A pesar de los importantes avances en las pruebas complementarias, el diagnóstico precoz sigue representando un gran desafío. Se revisa la experiencia de un servicio de urgencias en el diagnóstico de esta entidad, factores que pueden influir en la precocidad del diagnóstico y evolución intrahospitalaria.

Material y método: Se valoraron 86 pacientes diagnosticados de forma consecutiva en un centro hospitalario, analizando variables clínicas, pruebas diagnósticas realizadas, tratamiento y evolución hospitalaria.

Resultados: La edad promedio de los pacientes fue de $61,9 \pm 12,5$ años con predominio de varones (80\%). Tenían antecedentes de hipertensión el $65 \%$. El síntoma inicial dominante más frecuente era el dolor torácico $(58 \%)$. La impresión diagnóstica inicial fue de disección aórtica en el 56\%. La prueba complementaria más utilizada fue la TAC, que permitió la confirmación del diagnóstico en el 96\% de los casos en que fue realizada. Fueron intervenidos quirúrgicamente el $62 \%$. La mortalidad global intrahospitalaria fue del $28 \%$.

Conclusiones: Por la gran variabilidad de la presentación clínica es fundamental mantener un elevado índice de sospecha para el diagnóstico correcto. Los hallazgos de la exploración física clásicamente asociados a esta entidad no se presentan en una proporción considerable de casos. Un alto índice de sospecha clínica y la rápida realización de pruebas de imagen como la TAC, serían la principal estrategia para facilitar el diagnóstico.

PALABRAS CLAVE: Disección aórtica. Diagnóstico. Emergencia.

\begin{abstract}
Objective: Acute aortic dissection is a medical emergency associated with high rates of mortality. Despite recent diagnostic advances, prompt and accurate diagnosis is difficult. We present a review of the a medical emergency service experience to diagnosis, factors associated to prompt diagnosis and inhospital evolution.

Material and method: We studied 86 consecutive patients who had aortic dissections, to assess the presentation, management, and outcome.

Results: Mean age 61.9 12.5 years, $80 \%$ male. Hypertension was the most common predisposing factor (65\% of patients overall). Chest pain was the most common initial complaint (58\%). The initial clinical impression was aortic dissection in 56\% of patients overall. Computed tomography was diagnostic in $96 \%$ when used. $62 \%$ underwent surgery. Overall in-hospital mortality was $28 \%$

Conclusions: Acute aortic dissection presents with a wide range of manifestations. A high clinical index of suspicion and a prompt compu ted tomography should to improve recognition of aortic dissection.
\end{abstract}

KEY WORDS: Aortic dissection. Diagnosis. Emergency.

Muiño Miguez A, Villalba García MV, López González-Cobos C, Gómez Antúnez M, Ortiz Vega M, Ortiz Alonso J. Disección aórtica aguda. An Med Interna (Madrid) 2002; 19: 171-175.

\section{INTRODUCCIÓN}

La disección aórtica (DA) se caracteriza por la creación de una falsa luz en la capa media de la pared aórtica. Típicamente comienza con la formación de un desgarro en la íntima de la aorta, que expone la capa media subyacente al flujo sanguíneo, penetra disecándola y se extiende formando una falsa luz.
Desde su descripción inicial por Morgagni en 1761, continua representando un desafío su correcto manejo.

Su incidencia no es bien conocida, ya que se estima que más de un tercio de los pacientes no son diagnosticados nunca (1). Series de necropsias refieren incidencias de 5 a 10 por millón de habitantes y año (2), y estudios poblacionales hasta 30 casos por millón de habitantes y año (3-5). La incidencia se

Trabajo aceptado: 17 de enero de 2002

Correspondencia: A. Muiño Miguez. Hospital Gregorio Marañón. Departamento de Urgencias. C/ Doctor Esquerdo, 46.28007 Madrid. Télf. y Fax: 91 586 84 99. 
relaciona con la prevalencia de factores de riesgo como la hipertensión, el más influyente, edad avanzada y enfermedades de la pared aórtica (2).

El alto índice de sospecha es un elemento fundamental en el diagnóstico de la enfermedad, por lo variable de las manifestaciones iniciales y la trascendencia del retraso en el diagnóstico. Se describe una mortalidad, sin tratamiento, del uno por ciento cada hora durante las primeras cuarenta y ocho horas tras el comienzo de los síntomas (6). Entre los pacientes diagnosticados, en menos de la mitad el diagnóstico se realiza premorten (7).

Por otra parte, el desarrollo de pruebas complementarias no invasivas, ha representado un gran avance en las posibilidades de alcanzar un correcto diagnóstico.

Las disecciones aórticas se clasifican por la localiazción anatómica cuando se inicia. Se utilizan dos clasificaciones: La de DeBakey incluye tres tipos (tipo I, cuando la lesión se localiza en la aorta ascendente y descendente; tipo II, si la disección se limita a la aorta ascendente; tipo III, si la disección se localiza en la aorta torácica descendente, IIIa, o se extiende a la aorta abdominal y arterias ilíacas, IIIb) (8). La clasificación de Stanford considera dos tipos (tipo A, disección en la aorta ascendente con o sin afectación del arco o de la aorta descendente; tipo B, con afectación de la aorta torácica descendente) (9).

Se considera aguda la disección cuyos síntomas están presentes durante menos de dos semanas y crónica los demás casos. El período agudo se denomina así por su mayor morbilidad y mortalidad, estabilizándose habitualmente el paciente que supera esa fase (3).

Nuestro objetivo fue revisar la forma de presentación, diagnóstico y tratamiento de esta entidad en un servicio de urgencias de un hospital general.

\section{MATERIAL Y MÉTODOS}

Se revisaron 86 pacientes diagnosticados de DA aguda en un centro hospitalario entre 1993 y 1999. El diagnóstico fue confirmado en 53 mediante pruebas de imagen y cirugía $(62 \%)$ y en $33(38 \%)$ sólo por pruebas de imagen (TAC y/o ecocardiografía transtorácica y/o ecocardiografía transesofágica y/o angiografía). Se excluyeron los pacientes con DA secundaria a trauma.

Se registaron datos demográficos, factores de riesgo, clínica, pruebas diagnósticas, tratamiento realizado y evolución. La clasificación del tipo de disección se realizó siguiendo la clasificación de Standford. Se compararon los datos de los pacientes en los que se sospechó el diagnóstico tras la historia clínica con aquellos en que inicialmente se sospecharon otros procesos, los de tipo A y B de disección, así como de los supervivientes respecto a los fallecidos para intentar identificar factores pronósticos.

\section{RESULTADOS}

Los datos demográficos, antecedentes, síntomas iniciales y principales hallazgos del examen físico del conjunto de los pacientes y diferenciando disecciones tipo A y B se resumen en la tabla I.

El $80 \%$ de los pacientes eran varones. La edad fue en pro-

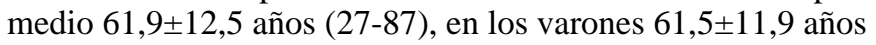

TABLA I

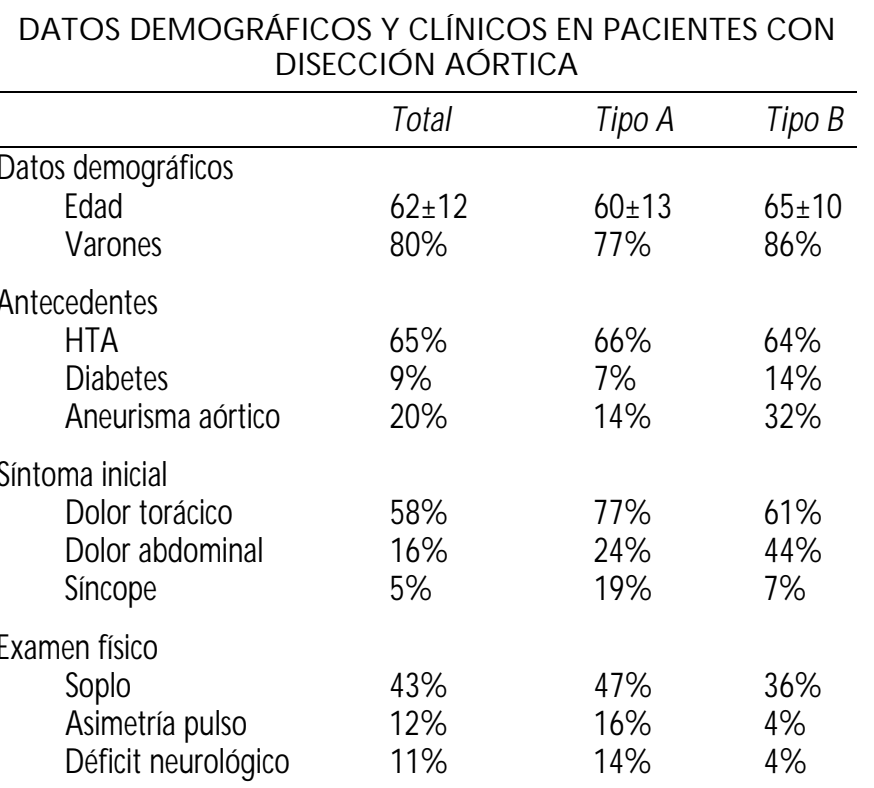

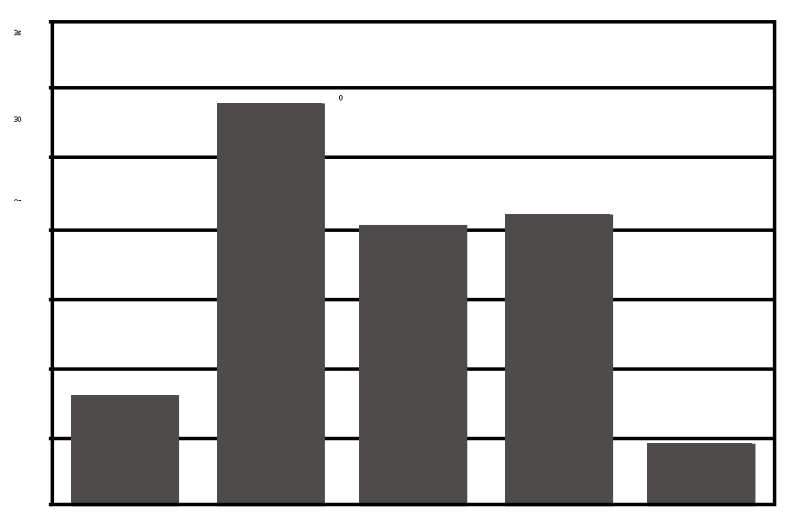

Fig. 1. Distribución grupos de edad.

y en las mujeres $63,5 \pm 15,1$ años (Fig. 1). El síntoma inicial más común fue dolor torácico $(58 \%)$, que era descrito como opresivo en el $43 \%$ y brusco en el $42 \%$. Se presentaban con dolor abdominal el 16\% (70\% epigástrico y $18 \%$ de localización lumbar) y síncope el 5\% (15\% los pacientes tenían síncope con o sin otros síntomas).

En el examen físico destacaban los siguientes hallazgos: el $43 \%$ presentaba soplo cardíaco, el $28 \%$ hipotensión, se constató asimetría de pulsos en el $12 \%$ y déficit neurológico el $11 \%$.

La sospecha clínica inicial, tras realizar anamnesis, exploración y ECG, era de disección en el $56 \%$ de los pacientes, mientras que en los demás casos se presumieron, en un principio, otros diagnósticos como cardiopatía isquémica $20 \%$, o cólico nefrítico $2 \%$. Los pacientes en los que no se estableció la sospecha diagnóstica inicial eran con más frecuencia disecciones tipo A, pero no existían diferencias significativas ni en edad, antecedentes, sintomatología, ni hallazgos de la exploración (Tabla II). 
TABLA ॥

HALLAZGOS EN PACIENTES CON DISECCIÓN SEGÚN IM PRESIÓ N CLÍNICA INICIAL

\begin{tabular}{lcc}
\hline Impresión clínica inicial & Disección & Otros \\
\hline Edad & $60 \pm 11$ & $64 \pm 13$ \\
Sexo V (\%) & $84 \%$ & $76 \%$ \\
Dolor torácico & $75 \%$ & $69 \%$ \\
Dolor abdominal & $37 \%$ & $24 \%$ \\
Disnea & $26 \%$ & $38 \%$ \\
Síncope & $14 \%$ & $17 \%$ \\
Soplo & $50 \%$ & $37 \%$ \\
Asimetría de pulso & $11 \%$ & $12 \%$ \\
Déficit neurológico & $9 \%$ & $12 \%$ \\
\hline
\end{tabular}

Entre las pruebas complementarias realizadas inicialmente en el servicio de urgencias, la radiografía de tórax, practicada al $68 \%$ de los pacientes, era normal en el $18 \%$ de los casos. Se reconoció ensanchamiento mediastínico o alteración del contorno aórtico en el $62 \%$ y derrame pleural en el $16 \%$.

El ecocardiograma transtorácico se realizó al $42 \%$ de los pacientes siendo diagnóstico en el $63 \%$ de los casos. Ecocardiograma transesofágico se efectuó al $28 \%$ de los casos, permitiendo el diagnóstico en el $87 \%$.

Se realizó TAC al $67 \%$ de los casos, permitiendo el diagnóstico en el $96 \%$.

La resonancia nuclear fue diagnóstica en los dos casos en los que se realizó.

Se recurrió a la arteriografía en el 30\% de los casos y en todos fue diagnóstica. La realización de unas pruebas de imagen u otras estuvo determinada por la disponibilidad y rapidez de realización de las mismas.

Fueron intervenidos quirúrgicamente el $62 \%$ de los pacientes.

Se objetivó taponamiento cardiaco en el 9,5\% de los pacientes. El 23\% de los pacientes con síncope presentaban un taponamiento frente al $7 \%$ (pacientes sin síncope que presentaron taponamiento).

La mortalidad intrahospitalaria fue del $28 \%$.

La disección era de tipo A en el $67 \%$ de los casos. Los pacientes con disección tipo B eran algo mayores ( $65 \pm 10$ años) y con más frecuencia varones $(86 \%)$ que los de tipo A $(60 \pm 13$ años, $77 \%$ varones), pero la diferencia no era significativa. No había diferencias en los antecedentes de enfermedad, excepto en el antecedente de aneurisma conocido (14\% en tipo A y $32 \%$ en tipo B). Fue más frecuente como síntoma de presentación el dolor torácico en el tipo A (77\% en el A, $61 \%$ en el B), el síncope (19\% en el A, $7 \%$ en el B) y la disnea ( $35 \%$ en el A, $25 \%$ en el B) y el dolor abdominal en el tipo B (44\% en el B, $24 \%$ en el A). Se objetivaron unas cifras de presión arterial (PA) inferiores en el grupo A (PAS: $116 \pm 34$ en el A y $162 \pm 39$ en el B; PAD: $59 \pm 28$ en el A y $90 \pm 24$ en el B, p<0,001) (Tabla III). Fueron intervenidos quirúrgicamente el $79 \%$ de los pacientes con disección tipo A y el $25 \%$ en los de tipo B. Los pacientes con disección tipo A en los que se decidió no intervenir, esta decisión se adoptó por edad avanzada y comorbilidad. Por el contrario en los pacientes con disección tipo B en los que no se siguió un manejo conservador, fue en aquellos en los que se preveía una evolución desfavorable por mayor diámetro de la aorta, progresión de la disección, mala perfusión de una rama aórtica distal, hipertensión incontrolable o dolor persistente.
TABLA III

HALLAZGOS EN DISECCIONES TIPOS A Y B

\begin{tabular}{lccc}
\hline & Tipo A & Tipo B & \\
\hline PAS $>150 \mathrm{mmHg}$ & $15 \%$ & $44 \%$ & $p<0,05$ \\
PAS $100-150 \mathrm{mmHg}$ & $50 \%$ & $48 \%$ & NS \\
PAS $<100 \mathrm{mmHg}$ & $35 \%$ & $7 \%$ & $p<0,01$ \\
Déficit de pulso & $16 \%$ & $4 \%$ & $p<0,05$ \\
\hline
\end{tabular}

La mortalidad fue del $34 \%$ en el tipo A y $14 \%$ en el tipo B $(\mathrm{p}<0,05)$ (Tabla IV).

Comparando las características de supervivientes y fallecidos, los pacientes hipotensos (PAS $<90)$ presentaron una mortalidad superior, aunque la diferencia no alcanzaba significación estadística ( $45 \%$ vs. $23 \%$, NS). No se asoció a mayor mortalidad en las disecciones de tipo A ni en las de tipo B la edad, sexo, antecedentes de hipertensión, ni patología aórtica previa conocida.

TABLA IV

TRATAM IENTO Y RESULTADOS EN DISECCIÓ N AÓ RTICA AGUDA

\begin{tabular}{lcccc}
\hline & \multicolumn{2}{c}{ Tipo A } & \multicolumn{2}{c}{ Tipo B } \\
\cline { 2 - 5 } & M édico & Q uirúrgico & M édico & Q uirúrgico \\
\hline № casos & 12 & 46 & 21 & 7 \\
M ortalidad & $50 \%$ & $30 \%$ & $5 \%$ & $43 \%$ \\
\hline
\end{tabular}

\section{DISCUSIÓN}

Dos factores influyen en la producción de una disección: un desencadenante y una debilidad estructural de la pared arterial. El estrés de la pared arterial puede deberse a hipertensión arterial o dilatación aórtica entre otras causas. La resistencia de la pared arterial se reduce con la edad, transtornos del sistema conectivo (síndrome de Marfan, Ehlers-Danlos) y el embarazo. La dilatación de la aorta o las presiones arteriales elevadas desgarran la íntima penetrando la sangre en la pared separando más sus capas (10).

La DA aguda no es una patología común, pero por su gavedad y las consecuencias del retraso en el diagnóstico, hacen imprescindible mantener un alto índice de sospecha basado en el conocimiento adecuado de esta entidad. La disección es un proceso dinámico que puede producirse en cualquier nivel de la aorta, por lo que la sintomatología puede diferir ampliamente de unos casos a otros, al presentar manifestaciones clínicas de isquemia en varios órganos aisladamente o en combinación.

Se puede reconocer un paciente prototipo caracterizado por ser varón en la séptima década de la vida, con antecedentes de hipertensión que presenta dolor torácico brusco. Pero como ya se ha indicado la variabilidad de estas características puede dificultar enormemente el diagnóstico, siendo en ocasiones un síncope sin dolor torácico, una isquemia de 
un miembro inferior o un déficit neurológico la única sintomatología.

El inicio brusco de dolor torácico severo retroesternal o interescapular, a veces referido como desgarro, y posterior progresión hacia abajo y hacia la espalda sería el cuadro típico. Si se produce ruptura de la adventicia y taponamiento puede ser rápidamente fatal o si se produce hemorragia masiva en mediastino o cavidad pleural. La disección puede comprimir u ocluir una rama de la aorta y producir isquemia aguda. La frecuencia descrita de las distintas localizaciones en que se produce isquemia es del $20 \%$ en extremidades, a nivel renal en el $15 \%$, miocardio $10 \%$, cerebro $5 \%$ y mesenterio o médula espinal 3\% (3).

Como otras casuísticas, la nuestra presenta un claro predominio de varones y una mayor frecuencia entre 50 y 75 años. El $18 \%$ de los casos no refería dolor torácico, en el rango de lo descrito en la literatura (14\%-55\%). La localización del dolor puede ayudar a predecir la localización de la dehiscencia de la íntima como en nuestra serie.

Presentaron síncope en nuestra serie un $15 \%$, y era el motivo de consulta en el 5\%. La presencia de síncope en los pacientes con disección se ha correlacionado con la ruptura de un proceso disecante en la cavidad pericárdica que produce taponamiento (7). En nuestra serie la presencia de taponamiento en pacientes con síncope era del $23 \%$, inferior a la de otras series (7).

Entre los hallazgos de la exploración más conocidos se encuentra el déficit de pulso como consecuencia de una oclusión vascular. Su frecuencia en nuestra casuística fue del $12 \%$, entre el 6 y $40 \%$ descrito en la literatura (11). Hay que recordar que en la población anciana la disminución de un pulso periférico es debido con una mayor frecuencia a enfermedad arteriosclerótica (7).

El uso de las pruebas complementarias de diagnóstico, que difiere notablemente de unas series a otras (12-14), se explica por la disponibilidad de las pruebas complementarias y la experiencia en su uso, variando de un centro a otro e incluso según el personal de guardia de unos días a otros. Aunque ninguna da certeza absoluta (15), la TAC se ha convertido en la prueba utilizada con más frecuencia y además con un elevado grado de precisión. La resonancia nuclear magnética, posiblemente más eficaz, es una prueba mucho menos accesible $(16,17)$.

La ecocardiografía juega también un lugar destacado en el diagnóstico. A su elevada eficacia, $75 \%$ en tipo A y $40 \%$ en tipo B con la transtorácica, se une la comodidad de poder ser realizada sin desplazar el paciente, por lo que es muchas veces la prueba preferida en pacientes inestables $(3,18)$. El ecocardiograma transesofágico es claramente más eficaz tanto para el diagnóstico como para el seguimeinto tras la intervención y detección de complicaciones. El esófago está en íntimo contacto con la aorta, por lo que su estudio es muy completo, con la limitación que impone la interposición de la tráquea y el bronquio principal izquierdo con la porción más alta de la aorta ascendente. Se describe una eficacia para esta técnica superior al 95\% (19).
Otras pruebas más simples como el electrocardiograma son de extraordinaria importancia en descartar otras patologías con las que es fácil que existan dudas como el infarto agudo de miocardio o la pericarditis. También es frecuente que existan dificultades de diagnóstico diferencial con enfermedad cardiaca valvular, crisis hipertensivas, embolismo pulmonar, cólico renal, colecistitis, etc. De todos ellos, es el infarto de miocardio el diagnóstico inicial incorrecto que se produce con más frecuencia.

El borramiento o ensanchamiento del botón aórtico, derrame pleural izquierdo, desviación traqueal, distancia mayor de $6 \mathrm{~mm}$ entre una calcificación intimal y el contorno externo de la pared aórtica, ensanchamiento mediastínico, ensanchamiento de la aorta ascendente o descendente y ensanchamiento de la línea paraespinal en la radiografía de tórax puede ser el signo de alarma inicial (20).

En nuestra serie fue correcta la sospecha diagnóstica inicial en el 55\%, similar a la descrita por otros autores (7). Se ha descrito que cuando el dolor afecta a tórax y espalda el diagnóstico se sospecha con más frecuencia que si el dolor afecta al abdomen (21). No obstante, en nuestra casuística, destaca la ausencia de peculiaridades remarcables del grupo en los que no se sospecha inicialmente disección y se produce una mayor demora en el diagnóstico. Esto nos induce a creer que el mayor o menor índice de sospecha por parte del médico que atiende inicialmente al paciente es el principal factor en la rapidez en el diagnóstico.

En series de principios de los años 70 se describía una mortalidad sin tratamiento de hasta el $70 \%$ a las 48 horas de inicio de los síntomas y hasta el $90 \%$ en la primera semana (6). El riesgo de ruptura fatal de la aorta ascendente o cayado aórtico es muy alto en las disecciones tipo A por lo que se recurre al tratamiento quirúrgico $(3,22,23)$.

$\mathrm{El}$ riesgo es menor en las de tipo $\mathrm{B}$, excepto en los casos de hematoma pleural izquierdo o periaórtico. El 70\% de los casos progresan a formas crónicas, por lo que se intenta el control médico, mediante el descenso de la presión arterial y la disminución del volumen sistólico para disminuir el estrés de la aorta (3). Después de la fase aguda, la ruptura se ha correlacionado con la edad avanzada, antecedente de enfermedad pulmonar obstructiva crónica y presión arterial elevada (24).

La mortalidad asociada a la intervención en las de tipo A se describe entre el 10-30\%, pero en los casos complicados por isquemia de algún territorio la mortalidad excede el $50 \%$. Las causas más frecuentes de mortalidad son la rotura aórtica, hemorragia, infarto de miocardio, afectación neurológica, insuficiencia cardiaca, insuficiencia renal, insuficiencia respiratoria e infección (25-28).

Por tanto debe existir un alto índice de sospecha en los médicos de urgencias ante esta entidad. Ante un paciente con antecedentes de hipertensión, que impresiona de gravedad, dolor torácico intenso de inicio súbito, con un soplo aórtico o un signo de oclusión vascular, debe plantearse el diagnóstico de disección aórtica y recordar la frecuencia de presentaciones atípicas o con poca expresión clínica. 


\section{Bibliografía}

1. Roberts CS, Roberts WC. Aortic dissection with the entrance tear in the descending thoracic aorta: analysis of 40 necropsy patients . Ann Surg 1991; 213: 356-68

2. Larson EW, Edwards WD. Risk factors for aortic dissection: a necropsy study of 161 cases. Am J Cardiol 1984; 53: 849-55.

3. Prêtre R, Von Segesser LK. Aortic dissection. Lancet 1997; 349: 1461-4.

4. American Heart Association . 1998 Heart and Stroke Statistical Update. Dallas, Tex: American Heart Association; 1998.

5. Meszaros I, Morocz J, Szlavi J, Schmidt J, Tornoci L, Nagy L, et al. Epidemiology and clinicpathology of aortic dissection. Chest 2000; 117: $1271-8$

6. Anagnopoulos CE, Prabhakar MJS, Kittle CF. Aortic dissections and dissecting aneurysms. Am J Cardiol 1972; 30: 263-73.

7. Spittell PC, Spittell JA, Joyce JW, Tajik AJ, Edwards WD, Schaff HV, Stanson AW. Clinical features and differential diagnosis of aortic dissection: Experience with 236 cases (1980 through 1990). Mayo Clin Proc 1993; 68: 642-51.

8. De Bakey M, Cooley D, Creech O. Surgical considerations of dissecting aneurysm of the aorta. Ann Surg 1955; 142: 586-612.

9. Daily PO, Truebold HW, Stinson EB, Wuerflein RD, Shumway NE. Management of acute aortic dissections. Ann Thorac Surg 1970; 10: 237-47.

10. Robicsek F, Thubrikar MJ. Hemodynamic considerations regarding the mechanism and prevention of aortic dissection .Ann Thorac Surg 1994; 58: 1247-53.

11. Hagan PG, Nienaber CA, Isselbacher EM, Bruckman D, Karavite DJ, Russman PL et al. The International Registry of ACute Aortic Dissection (IRAD). New Insights an Old Disease. JAMA 2000; 283: 897-903.

12. O'Gara PT, DeSanctis RW. acute aortic dissection and its variants: toward a common diagnostic and therapeutic approach . Circulation 1995; 92: 1376-8.

13. Cigarroa JE, Isselbacher EM, DeSanctis RW, Eagle KA. Diagnostic imagin in the evaluation of suspected aortic dissection : old standars and new directions. N Engl J Med 1993; 328: 35-43.

14. Crawford ES. The diagnosis and management of aortic dissection. JAMA 1990; 264: 2537-41.

15. Bansal RC, Chandrasekaran K, Ayala K, Smith D. Frequency and explanation of false negative diagnosis of aortic dissection by aortography and transesophageal echocardiography. J Am Coll Cardiol 1995; 25: 1393-401.
16. Nienaber CA, Spielmann RP, von Kodolitsch Y. Diagnosis of thoracic aortic dissection: magnetic resonance imaging versus transesophageal echocardiography. Circulation 1992; 85; 434-47.

17. Yamada T, Tada S, Harada J. Aortic dissection without intimal rupture: diagnosis with MR imaging and CT. Radiology 1988; 168: 347-52.

18. Armstrong WF, Bach DS, Carey LM, Froehlich J, Lowell M, Kazerooni EA. Clinical and echocardiographic findings in patients with suspected acute aortic dissection. Am Heart J 1998; 136: 1051-60.

19. Penco M, Paparoni S, Dagianti A, Fusilli C, Vitarelli A, De Remigis A, et al: Usefulness of transesophageal echocardiography in the assessment of aortic dissection. Am J Cardiol 2000; 86: 53G-6G.

20. Jagannath AS, Sos TA, Lockhart SH, Saddekni S, Sniderman KW. Aortic dissection: a statistical analysis of the usefulness of plain chest radiographic findings. Am J Radiol 1986; 147: 1123-6.

21. Sullivan PR, Wolfson AB, Leckey RD, Burke JL. Diagnosis of acute thoracic aortic dissection in the emergency department. Am J Emerg Med 2000; 18: 46-50.

22. Glower DD, Speier RH, White WD, Smith LR, Rankin JS, Wolfe WG. Management and long-term outcome of aortic dissection. Ann Surg 1991; 214: 31-41.

23. Heinemann M, Laas J, Karck M, Borst HG.Thoracic aortic aneurysms after acute type A aortic dissection: necessity for follow-up. Ann Thorac Surg 1990; 49: 580-4.

24. Juvonen T, Ergin MA, Galla JD, Lansman SL, McCullough JN, Bodian CA, et al. Risk factors for rupture of chronic type B dissections. J Thorac Cardiovasc Surg 1999; 117: 776-86.

25. Fann JI, Sarris GE, Mitchell RS. Treatment of patients with aortic dissection presenting with peripheral vascular complications. Ann Surg 1990; 212: 705-13

26. DeSanctis RW, Doroghazi RM, Austen WG, Buckley MJ. Aortic dissection. N Engl J Med 1987; 317: 1060-6.

27. Fattori R, Bacchi-Reggiani L, Bertaccini P, Napoli G, Fusco F, Lon M et al. Evolution of aortic dissection after surgical repair. Am J Cardiol 2000; 86: 868-72.

28. Lansman SL, McCullough JN, Nguyen KH, Spielvogel D, Klein JJ, Galla JD et al. Subtypes of acute aortic dissection. Ann Thorac Surg 1999; 67: 1975-8. 